Physical Sciences | Frédéric Grillot

\title{
Realising free-space communication with quantum cascade lasers
}

As cheap and accessible tools for transmitting private information, freely propagating light waves are highly desirable for parties operating secure However, the encoded information these waves contain can easily be lost due to unpredictable fluctuations in the Earth's atmosphere. In their research, Frédéric Grillot and Olivier Spitz at Télécom Paris have developed sophisticated new techniques to overcome this challenge. With specific lasers enciphering their the team's work could satterns, help to realise secure, globa scale networks, without the need for fibre optics. n today's fast-paced landscape of global communication, it is often critically important for many differen groups to exchange messages secur without any risk of eavesdroppers far, the most cutting-edge techniques for ensuring this level of security have often involved light waves transmitted through optical fibres, or radio waves sent through the air. However, these approaches face a number of drawbacks, particularly regarding the limited speeds at which large messages can be sent. Since the bandwidths available in optica fibres are strictly limited, they can quickly become saturated, leaving little room for information to propagate.

One emerging solution to this issue lies in 'free-space optics' (FSO), which operates using freely propagating light waves - but which doesn't require any

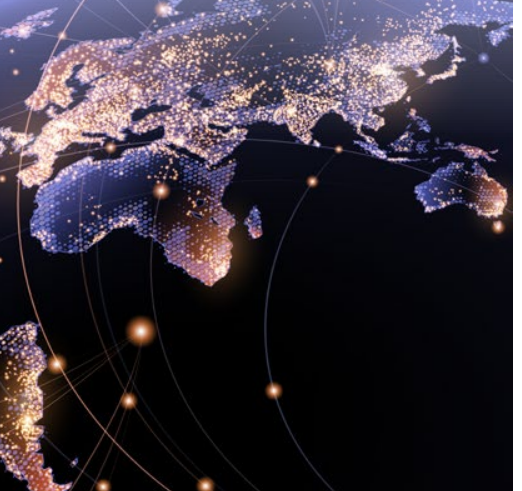

pre-existing infrastructure. In addition, these transmissions are not tied down by strict rules on which wavelengths can be Transmitted by particular parties. "Owing FSO has bocosteffective deployment. och radio frequencies, there is currently little regulation associated with FSO, so it can offer higher bandwidths." With far more room available for information to propagate, messages can be sent at significantly higher speeds.

Sending hidden messages this way takes advantage of the peculiarities of 'chaos,' meaning the laser output intensity is unpredictable and irregular in time. In this context, the term describes how timedependent patterns can appear to be in apparently random states of disorder but are actually governed by clearly dependent on their starting conditions If secret information is encodtitions. chaotic waveform, any receiving party which holds information about the naty of the chaos will be able to decipher it. On the other hand, an eavesdropper trying to intercept the message would be left with no way to untangle the information it contained.

\section{DIFFICULTIES WITH FLUCTUATIONS} FSO, it still faces an array of challenges before it can become a staple of secure global communication networks. Unlike the unchanging environment of a fibre optic cable, FSO requires light waves to pass through the turbulent conditions of Earth's atmosphere, introducing highly unpredictable fluctuations to the signa
Although this isn't the case for radio waves, these signals operate using far longer wavelengths, making them virtua

Without the right precautions in FSO transmission, any chaotic patterns known to two communicating parties will be distorted beyond recognition before signals reach their destination. Since just one, highly specific pattern can be used to decipher these messages, the information they contain would simply the influence of atmospheric distortion is not universal for all optical light waves.

"Chaos-based communication was originally proposed and demonstrated in electronic circuits, and then extend ooptical fibred systems," Dr Grillot to FSO is fundamentally restricted by 作 fog and scattering. Therefore, the operating wavelength is an important parameter that has to be chosen wisely to reduce the impact of the environmental parameters." In order to realise largescale networks with FSO, the research team now aims to develop technologies capable of producing wavelengths which can readily pass through the atmosphere while also generating chaotic patterns in real time.

\section{INTRODUCING QUANTUM}

CASCADE LASERS

Cist demonstrated in 1994, quantum cascade lasers (OCLs) are semicond djustability in the mid-infrared reg of the electromagnetic sectrum Since longer wavelengths are less vulnerable to atmospheric distortion, the light produced by OCLs is aready more desirable in FSO applications than applications than
optical wavelengths in the visible part of the spectrum. But mid-infrared light is particularly advantageous since Earth's atmosphere is highly transparent inside two distin 'windows': one for mid-infrared (MIR) wavelengths between 3 and 5 micron (UTIR) other for long 3 ave in frared (1) waver. 11 microns. (a)

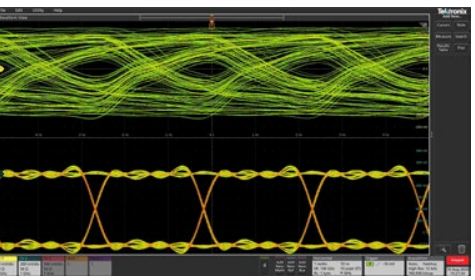

(b)

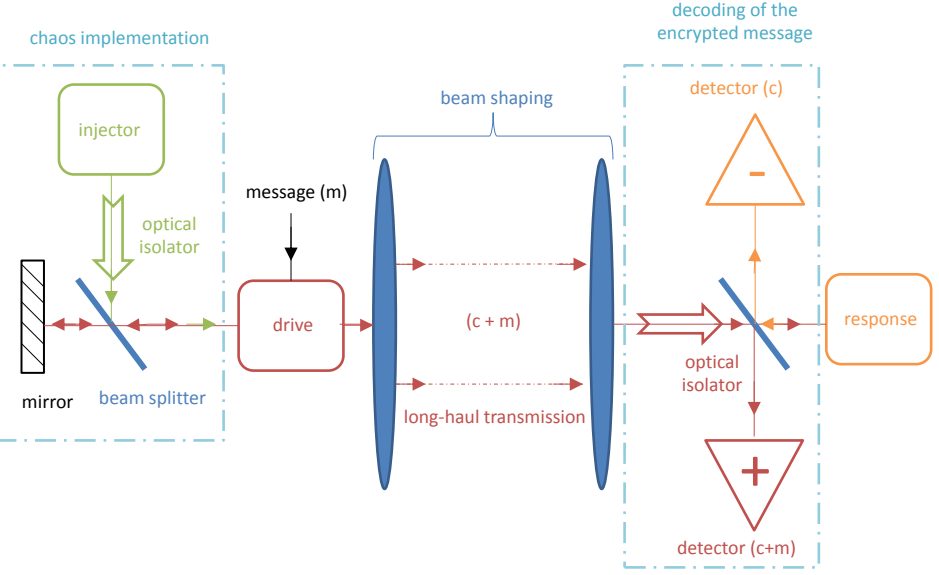

(a) Experimental eye diagram for the seed electrical signal (bottom time trace) and for the retrieved signal after transmission (top time trace). The data rate is $400 \mathrm{Mbi}$
(b) Schematic

"The MIR and LWIR optical regions emitted by QCLs are of prime interest for free-space communication," explains turbulence on the propagation path significantly deteriorates the optical signal. But not only is the absorption lower in the two transparency windows the signal distortion is also lower at the higher wavelengths emitted by these

QCLs have been demonstrating impressive abilities for some time. As early message at frequency of $300 \mathrm{MHz}$ over several hundreds of metres; at a longwave infrared wavelength of 9.3 microns. However, this feat required the quantum cascade laser to cool down, to minimise

ransmission, but at room temperature, and 4.65-micron wavelengths," Dr Grillot continues. "The versatility of this method, high-speed modulation up several dozens of $\mathrm{GHz}$, demonstrates that these lasers are poised to be the cornerstone of very high-speed free-space data transmission These capabilities essentially remove the need for bulky infrastructures like fibre optics and strictly regulated radio waves; instead, they open up the entife reques, to secure communica

\section{OBSERVING CHAOS IN THE MIR} In a 2016 study, Dr Grillot and his transmissions could definitively how such through the very first obsenvations of -in which a small
percentage of the percentage of the ght emitted by laser is reinjected any distortion. The technology has back into it. Typically, this technique advanced significantly in the years since is used to produce highly stable laser these initialexpeninents.

"More recently, investigations have beams, with few noticeable changes over time. In this case, however, the proportion $\mathrm{QCL}$ into a state of chaos. 


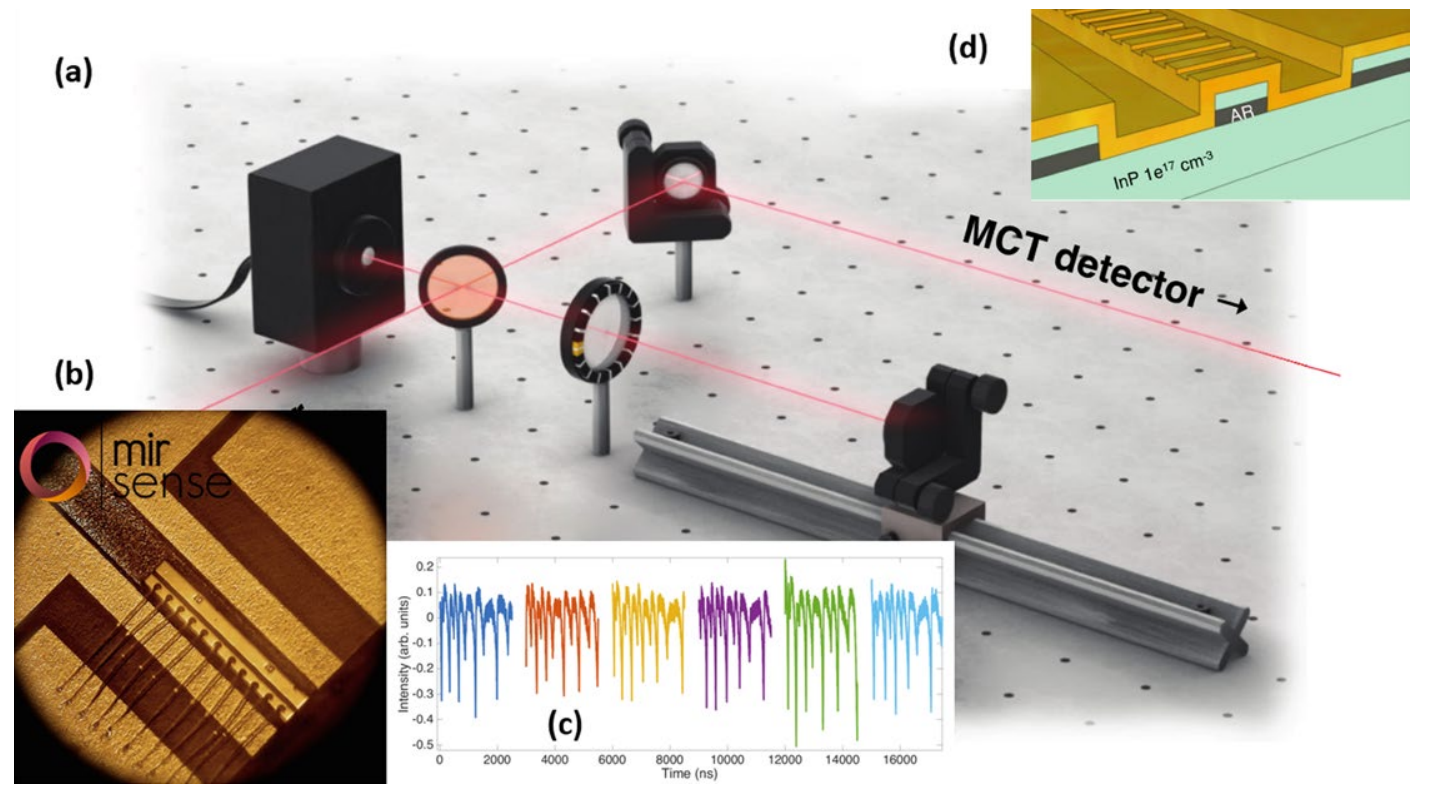

(a) Experimental setup with an external cavity for the optical feedback via a mirror; (b) Picture of
output of the quantum cascade laser; (d) Schematic of the quantum cascade laser structure.

This transformation occurred through a sequence of alterations to the nature of chaos in the light Once this sequence had played out, Dr Grillot's team observed that their OCL emitted lase pulses at completely unpredictable time intervals, enabling them to prove mathematically that chaos had been achieved. Their discovery was a significant breakthrough in the field of photonics as a whole, and for Dr Grillot, it enabled the next major step towards secure communication in space.

\section{SYNCHRONISED CHAOS}

For the purposes of FSO, QCLs can also ensure that the chaotic patterns which hide private messages can be maintained But in addition tranghiting must be able to generate this chass in the first place; and when the receiving laser picks up the signal it must be able to generate a synchronised pattern to decode the message. Therefore, synchronised chaos can be achieved through optical injection of the laser light between a drive $\mathrm{QCL}$ and a response $\mathrm{QCL}$

"At lower frequency rates, QCLs are of paramount importance for secure communication that can be obtained through chaos synchronisation," Dr Grillot describes. "The simplest way to generate a chaotic optical canier from a QCLis to feed part of its emitted light back into beyond which chas synchin between the drive and the response laser occurs." On the receiving end, the to the message can then be determined; and finally subtracted to leave behind a clear signal.

In their latest research, Drs Grillot and Spitz have explored how this technique could be improved even further through the technique of 'optical injection.'

spikes of signal that mimic the responses feurons in the brain. Overall, their towards secure, high-performance FSO, which could one day become widely accessible, and operational over expansive ranges. If achieved, this technology will pave the way for unbreakable communication networks which span the globe, and may even operate in space. Such attributes would

Their results represent an important advance towards secure, highperformance FSO using quantum cascade laser nonlinear photonics, which could one day become widely accessible, and operational over expansive ranges.

Here, the transmission laser is driven by an external $\mathrm{QCL}$ beam, instead of contain. Through their experiments, the team have used this approach to demonstrate transmission speeds ten times faster than those possible with optical feedback alone.

\section{REALISING LARGE NETWORKS}

From the insights gathered through their exploring an ever-expanding ar now applications including OCls which fie be highly desirable in a technological landscape where communicating parties are becoming increasingly concerned about their privacy.

As Dr Grillot concludes, "Several applications are envisioned including, but not limited to, campus-scaled networks: substitutions for fibre optics networks after disasters such as earthquakes or attacks, and private communication. The research team will now work towards improving the capabilities of the technology even further, bringing networks a step closer to reality. being fed back its own light - which significantly increases the range of possible frequencies its messages can

\section{Behind the Research} 畽

\section{Dr Frédéric} Grillot

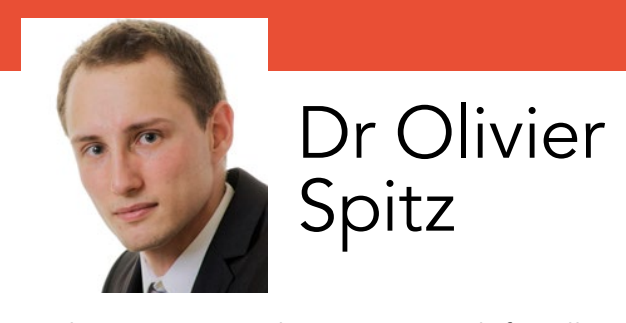

E: grillot@telecom-paris.fr T: +17867798708 W: https://perso.telecom-paristech.fr/grillot/

Research Objectives

Frédéric Grillot's research interests include semiconductor lasers, quantum confined devices, nonlinear photonics, highspeed optical communication and silicon photonics.

\section{Detail}

$\begin{array}{ll}\text { Frédéric Grillot } & \text { Olivier Spitz received his Ph.D. from } \\ \text { Télécom Paris, } 19 \text { Place Marguerite Perey } & \text { Université Paris-Saclay, France in } 2019\end{array}$ 91120 Palaiseau, France

Bio

Frédéric Grillot is a Full Professor at Télécom Paris and at the University of New-Mexico. He has published 106 journal articles and more than 200

contributions in major international
conferences. He is a Fellow Member of

the SPIE as well as a Senior Member of the OSA and the IEEE Photonics Society.
Olivier Spitz received his Ph.D. from
Université Paris-Saclay, France in 201 and is now Postdoctoral Researcher with Télécom Paris, working on applications of mid-infrared QCLs. He has been a visiting scholar at UCLA, USA, and at TU-Darmstadt, Germany. His research neuromorphic photonics.

• Direction Générale de l'Armement (DGA) interests include non-linear dynami

\section{- Agence Nationale de la Recherche} (ANR)

European Office of Aerospace Researc

\section{Collaborators}

Telécom Paris (Dr O. Spitz); mirSense (Dr M. Carras); UC Los Angeles ProfW. Elsaesser)

\section{References}

-Spitz O, Herdt A, Maisons G, et al. (2019, Sept). High frequency dynamics in quantum cascade lasers: a infrared. In Applications of Lasers for Sensing mid Space Communications (pp. LWAB-2). Optical Society of America.

- Spitz O, Wu J, Carras M, et al. (2020, May). Excitability in mid-infrared quantum cascade lasers: from communication
jamming to neuromorphic photonics. In 2020 Conference on Lasers and Electro-Optics (CLEO) (pp. 1-2). IEEE. - Grillot F, Spitz O, Herdt A, et al. (2020, Jan). Towards private optical communications with mid-infrared chaotic light. In Quantum Sensing and Nano Electronics and Photonics XVII (Nol. 11288, p. 112881P). International Society for Optics and Photonics.

- Spitz O, Wu J, Herdt A, et al. (2019). Investigation of chaotic and spiking dynamics in mid-infrared quantum cascade lasers operating continuous-waves and under current modulation. J Sel Top Quantum Electron 25(6): :1-11:

Jumpertz L, Schires K, Carras M, et al. (2016). Chaotic light at mid-infrared wavelength. Light Sci App/5,

\section{Personal Response}

How will the nature of our communication networks change if the findings of your research sources in the MIR/LWIR or the use of chaos private
transmission in free-space optical communication will facilitate the high-speed long-haul data transmission as well as the implementation of a reliable level of privacy for such transmitted data. The main advantages of this method are a high resistance to temperature packaging. All in all, this unlocks the capability of a wide and uncharted optical domain for
an ever-growing telecom market.

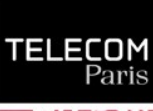

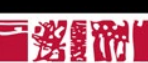

II The permanent use of quantum cascade laser external atmospheric perturbations, a physical-layer optical sources that come in compact and room--sa 